\title{
Improving the Predictive Accuracy for Pre-eclampsia by Combining Demographic and Socio-economic Risk Factors with Biochemical Indicators
}

\author{
Ana Daneva Markova*, Goran Dimitrov, Gligor Tofoski, Jadranka Georgievska, Elena Dzikova, Vesna Livrinova, Ivo Kjaev \\ and Marijana Filipovska \\ University clinic of obstetrics and gynecology, Skopje, Macedonia
}

\section{Abstract}

Publication History:

Objective: Since prevention of pre-eclampsia is not possible, the target should be to estimate the severity of the disease that will provide intensive supervision during the further course of pregnancy. So, the aim of this study is to improve the predictive accuracy for pre-aclampsia by defining the demographic and socio-economic characteristicsofpregnantwomen with pre-eclampsiain Macedoniacombined withcertainbiochemicalindicators.

Methods and materials: The data used in this study are collected from a case control study which was conductedat the University Clinic of Gynecology and Obstetrics, University "Ss. Cyril and Methodius" in Skopje, Macedonia. The study included 50 pregnant women with singleton pregnancies between 28 and 40 gestational weeks hospitalized with symptoms of pre-eclampsia, determined by standard clinical laboratory analysis. The control group consisted of 50 normotensive pregnant women of the same gestational age.

After obtaining consent for participation in the examination of every pregnant woman, a detailed history was taken, cardiotocography and ultrasound biometry were preformed. Also a blood sample was drawn to determine serum concentrations of appropriate markers and clinical laboratory testing to assess the severity of pre-eclampsia (moderate or severe). Inflammatory cytokines IL-10 was analyzed from peripheral blood $1-1,5 \mathrm{ml}$ by enzymatic amplification chemiluminescence on the device Immulite 1000, Siemens Healthcare Diagnostics, USA.

The data were digitized and all statistical tests were performed using SPSS version 13.0. Logistic regression analysis (Binary Logistic Regression) was used to determine the predictive value of the different parameters for the occurrence of severe preeclampsia. Rates of probability - odds ratios (OR) and 95\% Confidence Intervals (CI) were calculated in order to quantify independent associations.

Results: Analysis applied in this study for several of the demographic and clinical risk factors for pre-eclapsia showed that elevated systolic blood pressure of $160 \mathrm{mmHg}$ or higher, diastolic blood pressure of $100 \mathrm{mmHg}$ or higher, pregnancy at older age than 35 years as is nulliparity are associated with highly significant risk for developing severe form of pre-eclampsia. Other risk factors examined in this survey such as duration of gestation, BMI, number of pregnancies, previous pregnancy with pre-eclampsia, diabetes and smoking status according to the results of this study, are risk factors that insignificantly increase the risk for severe form of pre-eclapmsia. As for the biochemical indicators, reduced serum concentrations of IL10 are statistically significantly associated with the development of severe form of preeclampsia in pregnant women. IL10 was also found to be negatively correlated with proteinuria, and positively correlated with blood platelets. Significantly higher concentration of IL10 was confirmed in patients with higher number of platelets in the blood, and vice versa. On the other hand, the serum concentration of IL10 was significantly lower in patients with higher amount of proteins in the urine, and vice versa.

Conclusions: Examination of clinical risk factors combined with biochemical markers can improve the predictive success of pre-eclampsia and has important clinical values in improving the prognosis of pregnant women and fetuses.

\section{Introduction}

Pre-eclampsia, defined as the onset of hypertension and the presence of protein in the urine at $>20$ weeks of gestation in a previously normotensive woman, is a pregnancy complication that is still one of the leading causes of death and disability of both mother and babies. Pre-eclampsia occurs in 5-8\% of pregnancies in developed countries.

Risk factors for pre-eclampsia that have been identified in previous studies include: both young and old maternal age, high BMI, prior pregnancy with pre-eclampsia, excessive weight gain during pregnancy, nulliparity, chronic hypertension, low socioeconomic status, prolonged birth interval, race and ethnicity, genetic predisposition,
Received: December 31, 2018

Accepted: March 02, 2019

Published: March 04, 2019

\section{Keywords:}

Inflammation, Cytokines, Preeeclampsia, Biochemical markers, Neonatal outcomes environmental and even seasonal influences. Ironically, although smoking during pregnancycausesvariousadversepregnancyoutcomes, whenitcomestopre-eclampsiaandhypertensivedisordersinpregnancy, many studies have shown that it is associated with reduced risk.

${ }^{*}$ Corresponding Author: Prof. Ana Daneva Markova, University clinic of obstetrics and gynecology, Skopje, Macedonia; E-mail: anadaneva@yahoo.com

Citation: Markova AD, Dimitrov G, Tofoski G, Georgievska J, Dzikova E, et al. (2019) Improving the Predictive Accuracy for Pre-eclampsia by Combining Demographic and Socio-economic Risk Factors with Biochemical Indicators. Int J Pediatr Neonat Care 5: 148. doi: https://doi.org/10.15344/2455-2364/2019/148

Copyright: (C) 2019 Markova et al. This is an open-access article distributed under the terms of the Creative Commons Attribution License, which permits unrestricted use, distribution, and reproduction in any medium, provided the original author and source are credited. 
Citation: Markova AD, Dimitrov G, Tofoski G, Georgievska J, Dzikova E, et al. (2019) Improving the Predictive Accuracy for Pre-eclampsia by Combining Demographic and Socio-economic Risk Factors with Biochemical Indicators. Int J Pediatr Neonat Care 5: 148. doi: https://doi.org/10.15344/2455-2364/2019/148

Page 2 of 6

The role of immune mechanisms contributing to the development of a normal pregnancy is widely discussed. Their involvement in the pathogenesis of pregnancy complications, such as preeclampsia, was also noted. The analysis of the scientific literature reveals conclusion that many aspects of the pathogenesis of preeclampsia are related with systemic inflammatory response syndrome with the development of a destructive inflammatory process, immune disorders, and the imbalance of cytokine regulation of gestation processes.Studies showed that in pregnancy complicated by preeclampsia, cytokine levels essentially change compared with the respective levels in physiological pregnancy. Thus, even a moderate form of preeclampsia shows directional change, i.e., elevated levels of pro- and antiinflammatory cytokines, with the exception of IL-10, wherein a downward trend in severe preeclampsia is recorded.

We carried out this study to estimate the risk of developing different forms of pre-eclampsia for each previously known demographic and clinical risk factor as to evaluate the relationship between the formation of anti-inflammatory IL10cytokine and several other biochemical indicators of moderate and severe preeclampsia.This will provide an evidence base from which healthcare professionals can assess each pregnant woman's risk of pre-eclampsia at her first antenatal visit and arrange her antenatal care according to need.

\section{Prediction for Severe / Non-severE Pre-eclampsia}

Uni-variant Logistic Regression Analysis for determination of the predictive role of certain socio - demographic, clinical and biochemical parameters for severe pre-eclampsia.

\section{Maternal age}

Maternal age of patients with non-severe and severe pre-eclampsia was analyzed into two categories: older than 35 and younger than 35 years. The results showed that $16 \%$ of the patients with nonsevere form of pre-eclampsia and $52 \%$ of the patients with severe form of preeclampsia were older than 35 years. The statistical analysis confirmed that pregnant women older than 35 years, highly significantly, have severe form of preeclampsia $(p=0,007)$.
The age of the respondents analyzed as continuous variable has confirmed itself as highly significant predictor for severe form of preeclampsia $(p=0,004)$. Advancing the age for another year increases the probability for getting severe form of pre-eclampsia during the pregnancy for $26,3 \%(95,0 \%$ CI 1.08 - 1,478) (Table 1).

The age analysis as categorical variable in two age groups (older and younger than 35 years), have shown that pregnant women older than 35 years are in 5,687 times $(95,0 \%$ CI 1,510 - 21,424) bigger risk from the pregnant women aged 35 and younger to develop severe form of preeclampsia (Table 2).

\section{Gestation}

The probability for getting severe form of preeclampsia insignificantly decreases with the increase of the gestation length of the pregnant women $(\mathrm{p}=0,271)$. If the pregnancy continues for one more gestational week, the chance for getting severe form of preeclampsia decreases by $8,8 \%(95,0 \%$ CI 0,775 - 1,74) (Table 3$)$.

\section{BMI}

Although studies from developed countries show that high prepregnancy body mass index is associated with increased risk of preeclampsia, for level of significance of $\mathrm{p}=0,05$, the results of this survey have confirmed that the value of the BMI is insignificant factor for severe pre-eclampsia $(\mathrm{p}=0,059)$ (Table 4$)$.

\section{Nulliparity}

The nulliparity represents highly significant risk factor for severe form of pre-eclampsia $(\mathrm{p}=0,006)$. Pregnant women without history for previous delivery are in 5,63 times $(95,0 \%$ CI 1,648-19,232) higher risk than the pregnant women who previously gave birth for getting a severe form of preeclampsia (Table 5).

\section{Number of pregnancies}

The number of pregnancies is insignificant risk factor for severe form of pre-eclampsia $(\mathrm{p}=0,882)$. Pregnant women with two pregnancies

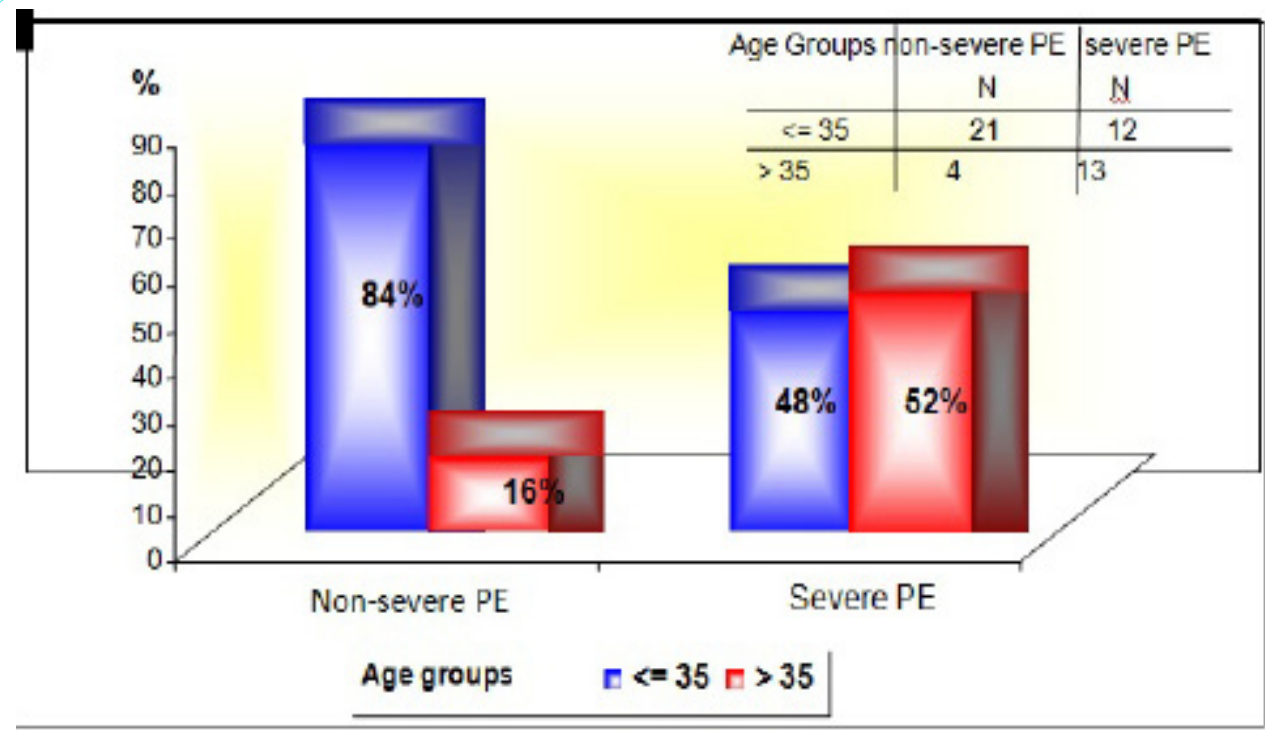

Figure 1: Age groups - non-severe and severe PE.

Pearson Chi-square: $7,21 \mathrm{df}=1, \mathrm{p}=0,007^{\star \star} \mathrm{p}<0,01$. 
Citation: Markova AD, Dimitrov G, Tofoski G, Georgievska J, Dzikova E, et al. (2019) Improving the Predictive Accuracy for Pre-eclampsia by Combining Demographic and Socio-economic Risk Factors with Biochemical Indicators. Int J Pediatr Neonat Care 5: 148. doi: https://doi.org/10.15344/2455-2364/2019/148

Page 3 of 6

have 0,857 times $(95,0 \%$ CI $0,111-6,617)$ insignificantly smaller chance than the ones with one pregnancy to develop severe form of pre-eclampsia (Table 6).

\section{Previous preeclampsia}

The results of our survey did not show that previous pre-eclampsia significantly increases the chance for getting severe form of preeclampsia $(p=0,215)$. Pregnant women with history for previous preeclampsia have 3,028 times insignificantly larger probability from those with negative history for previous pre-eclampsia to develop severe form of preeclampsia (Table 7)

\section{Smoking status}

Smoking cigarettes insignificantly increases the risk for severe preeclampsia $(p=0,096)$. Pregnant women that are smokers are in 3,15 times insignificantly higher risk than the pregnant women that are non-smokers for getting severe pre-eclampsia (Table 8).

\section{Diabetes mellitus}

Diabetes type 1, type 2 or gestation diabetes are insignificantly associated with severe form of pre-eclampsia in the pregnancy $(\mathrm{p}=0,364)$. The chances for getting a severe pre-eclampsia increases 2,3

\begin{tabular}{|l|l|l|l|l|l|l|l|}
\hline Variable & B & S.E. & Wald & Sig. & $\operatorname{Exp}(\mathrm{B})$ & $95,0 \%$ CI for $\operatorname{Exp}(\mathrm{B})$ \\
\hline Age & 0,234 & 0,080 & 8,510 & $0,004^{* *}$ & 1,263 & 1,080 & 1,478 \\
\hline Constant & $-7,510$ & 2,603 & 8,323 & 0,004 & 0,001 & & \\
\hline
\end{tabular}

Table 1: Uni- Variant Logistic Regression Analysis - the meaning of age in the prediction of severe preeclampsia. Dependent variable - severe preeclampsia/ non-severe preeclampsia ${ }^{\star *} \mathrm{p}<0,01$.

\begin{tabular}{|l|l|l|l|l|l|l|l|}
\hline Variable & B & S.E. & Wald & Sig. & $\operatorname{Exp}(B)$ & \multicolumn{2}{|l|}{$95,0 \%$ CI for Exp (B) } \\
\hline Age $>35$ & 1,738234 & 0,677 & 6,599 & $0,01^{* *}$ & 5,687 & 1,510 & 21,424 \\
\hline Constant & $-0,56$ & 0,362 & 2,391 & 0,122 & 0,571 & & \\
\hline
\end{tabular}

Table 2: Uni- Variant Logistic Regression Analysis - age over 35 years for prediction of severe preeclampsia. Dependent variable - severe preeclampsia/ medium preeclampsia ${ }^{* *} \mathrm{p}<0,01$.

\begin{tabular}{|l|l|l|l|l|l|l|l|}
\hline variable & B & S.E. & Wald & Sig. & $\operatorname{Exp}(B)$ & \multicolumn{2}{|l|}{$95,0 \%$ CI for $\operatorname{Exp}(\mathrm{B})$} \\
\hline gestation & $-0,092$ & 0,083 & 1,211 & 0,271 & 0,912 & 0,775 & 1,74 \\
\hline Constant & 3,211 & 2,935 & 1,197 & 0,274 & 24,796 & & \\
\hline
\end{tabular}

Table 3: Uni- Variant Logistic Regression Analysis - gestational age in prediction for severe preeclampsia.

Dependent variable - severe eclampsia/ medium preeclampsia.

\begin{tabular}{|l|l|l|l|l|l|l|l|}
\hline Variable & B & S.E. & Wald & Sig. & Exp(B) & \multicolumn{2}{|l|}{$95,0 \%$ CI forExp (B) } \\
\hline BMI & 0,131 & 0,070 & 3,562 & 0,059 & 1,140 & 0,995 & 1,307 \\
\hline Constant & $-4,505$ & 2,407 & 3,504 & 0,061 & 0,011 & & \\
\hline
\end{tabular}

Table 4: Uni- Variant Logistic Regression Analysis - BMI in prediction of severe preeclampsia.

Dependent variable - severe preeclampsia/ medium preeclampsia.

\begin{tabular}{|l|l|l|l|l|l|l|l|}
\hline Variable & B & S.E. & Wald & Sig. & \multicolumn{2}{|l|}{$\operatorname{Exp}(B)$} & \multicolumn{2}{|l|}{$95,0 \%$ CI forExp(B) } \\
\hline Nulliparity & 1,728 & 0,627 & 7,600 & 0,006 & 5,630 & 1,648 & 19,23 \\
\hline Constant & $-0,981$ & 0,479 & 4,198 & 0,04 & 0,375 & & \\
\hline
\end{tabular}

Table 5: Uni- Variant Logistic Regression Analysis - zero parity in prediction of severe preeclampsia. Dependent variable - severe preeclampsia/ medium preeclampsia ${ }^{\star *} \mathrm{p}<0,01$.

\begin{tabular}{|l|l|l|l|l|l|l|l|}
\hline Variable & B & S.E. & Wald & Sig. & Exp(B) & \multicolumn{2}{|l|}{$95,0 \%$ CI forExp (B) } \\
\hline N of preg. & $-0,154$ & 1,043 & 0,022 & 0,882 & 0,857 & 0,111 & 6,617 \\
\hline Constant & $-1,099$ & 0,667 & 2,716 & 0,099 & 0,333 & & \\
\hline
\end{tabular}

Table 6: Uni- Variant Logistic Regression Analysis - The number od pregnancies in prediction of severe preeclampsia. Dependent variable - severe eclampsia/ non-severe preeclampsia.

\begin{tabular}{|l|l|l|l|l|l|l|l|}
\hline Variable & B & S.E. & Wald & Sig. & \multicolumn{2}{|l|}{$\operatorname{Exp}(\mathrm{B})$} & \multicolumn{2}{|c|}{$95,0 \%$ CI forExp (B) } \\
\hline Previous PE & 1,10 & 0,892 & 1,540 & 0,215 & 3,028 & 0,527 & 17,394 \\
\hline Constant & 0,191 & 0,310 & 0,380 & 0,538 & 0,828 & & \\
\hline
\end{tabular}

Table 7: Uni - Variant Logistic Regression Analysis -Previous preeclampsia in prediction of severe preeclampsia. Dependent variable - severe preeclampsia/ non-severe preeclampsia 
Citation: Markova AD, Dimitrov G, Tofoski G, Georgievska J, Dzikova E, et al. (2019) Improving the Predictive Accuracy for Pre-eclampsia by Combining Demographic and Socio-economic Risk Factors with Biochemical Indicators. Int J Pediatr Neonat Care 5: 148. doi: https://doi.org/10.15344/2455-2364/2019/148

Page 4 of 6

times insignificantly for the pregnant women with diabetes mellitus compared with the pregnant women without diabetes (Table 9).

\section{Systolic blood pressure}

Systolic blood pressure form $160 \mathrm{mmHg}$ and higher is measured in $20 \%$ of the patients in the group with non-severe form of eclampsia, and in $92 \%$ of the patients from the group with severe form. The difference in the distribution of respondents with values of systolic blood pressure higher and lower than $160 \mathrm{mmHg}$ is statistically highly significant $(\mathrm{p}=0,01)$ (Figure 2).

Systolic blood pressure analyzed as continuous variable has confirmed itself as highly significant predictor for severe preeclampsia in pregnancy $(\mathrm{p}=0,001)$. The increasing of systolic blood pressure for $1 \mathrm{mmHg}(95,0 \%$ CI1,009-1,423) increases the probability for $25 \%$ for severe pre-eclampsia (Table 9).

Pregnant women who have systolic blood pressure $160 \mathrm{mmHg}$ and higher have 46 times (95,0\% CI8,027-1,423) significantly higher chance than pregnant women with systolic blood pressure lower than $160 \mathrm{mmHg}$ to develop severe form of pre-eclampsia (Table 10).

\section{Diastolic blood pressure}

Diastolic blood pressure of $100 \mathrm{mmHg}$ and higher more often had the respondents from the group with severe pre-eclampsia compared with the ones from the group with non-severe preeclampsia ( $88 \%$ vs. 44\%) (Figure 3).

Diastolic blood pressure highly significant can predict phenomenon of severe preeclampsia in pregnancy $(p=0,000)$. The increasing of the diastolic blood pressure for $1 \mathrm{mmHg}$ increases the probability for severe pre-eclampsia for $29,8 \%(95,0 \%$ CI1,129-1,492) (Table 11).

Pregnant women who have diastolic blood pressure $100 \mathrm{mmHg}$ and higher have 11 times significantly higher chance than pregnant women with diastolic blood pressure lower than $100 \mathrm{mHg}$ to develop severe form of eclampsia (Table 12).

\begin{tabular}{|l|l|l|l|l|l|l|l|}
\hline variable & B & S.E. & Wald & Sig. & \multicolumn{2}{|l|}{$\operatorname{Exp}(\mathrm{B})$} & \multicolumn{2}{|l|}{$95,0 \%$ CI forExp (B) } \\
\hline smoker & 1,147 & 0,689 & 2,769 & 0,096 & 3,150 & 0,815 & 12,168 \\
\hline Constant & $-0,336$ & 0,338 & 0,991 & 0,320 & 0,714 & & \\
\hline
\end{tabular}

Table 8: Uni- Variant Logistic Regression Analysis -Previous preeclampsia in prediction of severe eclampsia. Dependent variable - severe eclampsia/ non-severe eclampsia.

\begin{tabular}{|l|l|l|l|l|l|l|l|}
\hline Variable & B & S.E. & Wald & Sig. & \multicolumn{2}{|l|}{$\operatorname{Exp}(\mathrm{B})$} & \multicolumn{2}{|l|}{$95,0 \%$ CI forEx (B) } \\
\hline Diabetes & 0,833 & 0,918 & 0,822 & 0,364 & 2,300 & 0,380 & 13,915 \\
\hline Constant & $-0,140$ & 0,306 & 0,209 & 0,648 & 0,870 & & \\
\hline
\end{tabular}

Table 9: Uni- Variant Logistic Regression Analysis -Diabetes mellitus in prediction of severe preeclampsia.

Dependent variable - severe eclampsia/ non-severe preeclampsia.

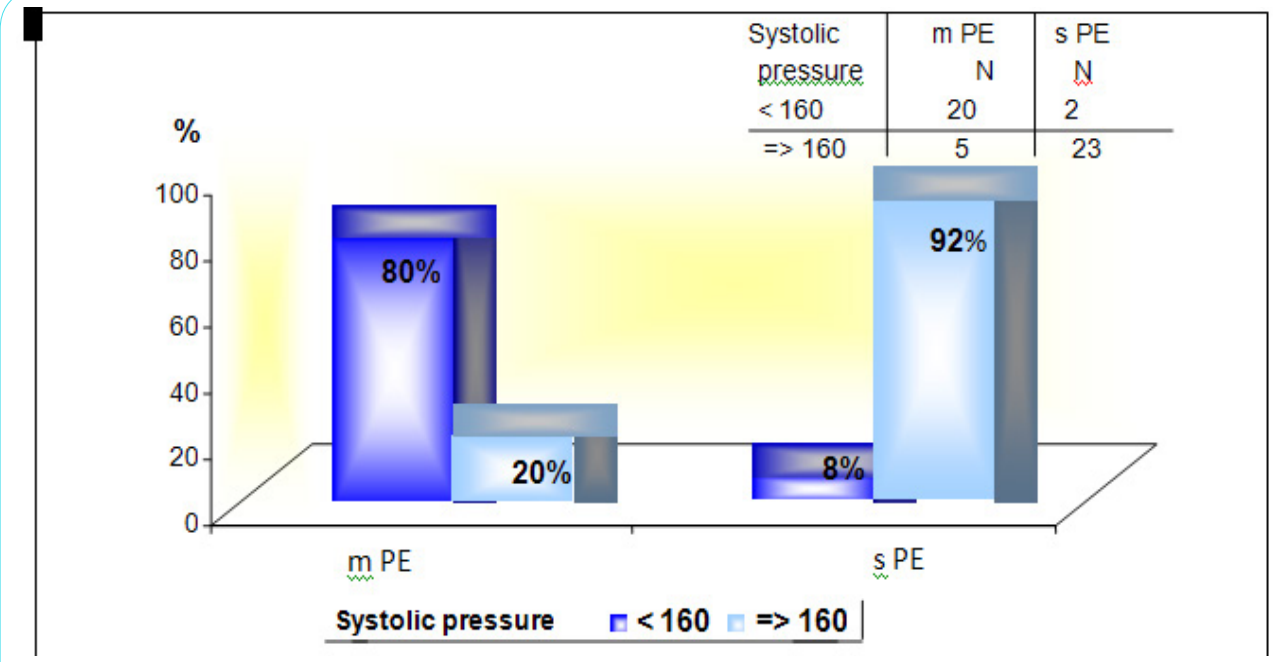

Figure 2: Systolic blood pressure - non-severe and severe PE.

Pearson Chi-square: $26,29 \mathrm{df}=1, \mathrm{p}=0,000^{* *} \mathrm{p}<0,01$.

\begin{tabular}{|l|l|l|l|l|l|l|c|}
\hline Variable & B & S.E. & Wald & Sig. & Exp(B) & \multicolumn{2}{|l|}{$95,0 \%$ CI for Exp (B) } \\
\hline Systolic pressure & 0,224 & 0,066 & 11,464 & $0,001^{\star *}$ & 1,250 & 1,099 & 1,423 \\
\hline Constant & $-35,524$ & 10,558 & 11,321 & 0,001 & 0,000 & & \\
\hline
\end{tabular}

Table 10: Uni- Variant Logistic Regression Analysis -Systolic blood pressure in prediction of severe eclampsia. Dependent variable - severe eclampsia/ non-severe eclampsia ${ }^{* *} \mathrm{p}<0,01$. 
Citation: Markova AD, Dimitrov G, Tofoski G, Georgievska J, Dzikova E, et al. (2019) Improving the Predictive Accuracy for Pre-eclampsia by Combining Demographic and Socio-economic Risk Factors with Biochemical Indicators. Int J Pediatr Neonat Care 5: 148. doi: https://doi.org/10.15344/2455-2364/2019/148

Page 5 of 6

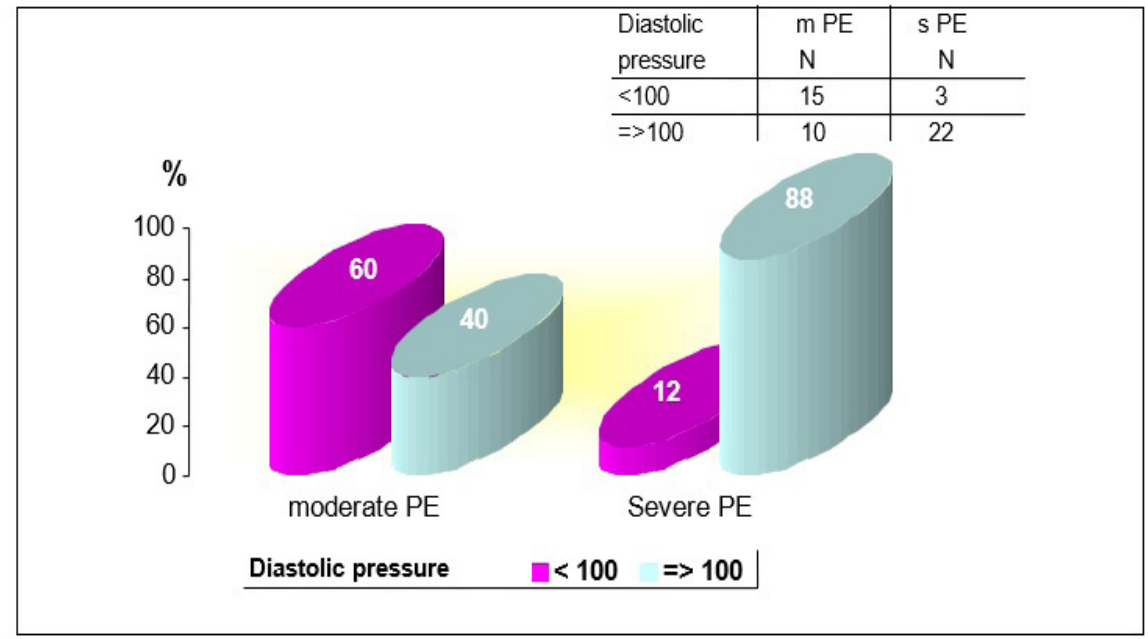

Figure 3: Diastolic blood pressure - medium and severe PE.

Pearson Chi-square: $12,5, \mathrm{df}=1, \mathrm{p}=0,0004^{\star *} \mathrm{p}<0,01$

\begin{tabular}{|l|l|l|l|l|l|l|l|}
\hline Variable & B & S.E. & Wald & Sig. & \multicolumn{2}{|l|}{$\operatorname{Exp(B)}$} & \multicolumn{2}{l|}{$95,0 \%$ CI forExp (B) } \\
\hline Diastolic pressure & 0,261 & 0,071 & 13,456 & $0,000^{* *}$ & 1,298 & 1,129 & 1,492 \\
\hline Constant & $-26,505$ & 7,158 & 13,710 & 0,001 & 0,000 & \\
\hline
\end{tabular}

Table 11: Uni- Variant Logistic Regression Analysis -Diastolic blood pressure higher than 160mmHg in prediction of severe eclampsia.

Dependent variable - severe eclampsia/ medium eclampsia ${ }^{* *} \mathrm{p}<0,01$

\begin{tabular}{|l|l|l|l|l|l|l|l|}
\hline Variable & B & S.E. & Wald & Sig. & \multicolumn{2}{|c|}{$\operatorname{Exp(B)}$} & \multicolumn{2}{|l|}{$95,0 \%$ CI for Exp (B) } \\
\hline DyP $=>100$ & 2,398 & 0,739 & 10,541 & $0,001^{\star *}$ & 11,0 & 2,587 & 46,779 \\
\hline Constant & $-1,609$ & 0,632 & 6,476 & 0,001 & 0,2 & & \\
\hline
\end{tabular}

Table 12: Uni- Variant Logistic Regression Analysis -Diastolic blood pressure higher than 160mmHg in prediction of severe preeclampsia.

Dependent variable - severe eclampsia/ medium eclampsia ${ }^{* *} \mathrm{p}<0,01$

\begin{tabular}{|l|l|l|l|l|l|l|c|}
\hline Variable & B & S.E. & Wald & Sig. & \multicolumn{2}{|l|}{$\operatorname{Exp(B)}$} & \multicolumn{2}{|l|}{$95,0 \%$ CI for Exp (B) } \\
\hline IL-10 & $-2,267$ & 0,958 & 5,598 & $0,018^{\star *}$ & 0,104 & 0,016 & 46,779 \\
\hline Constant & 3,820 & 1,235 & 9,565 & 0,002 & 45,612 & & \\
\hline
\end{tabular}

Table 13: Logistic regression analysis for the factor's predictors of severe preeclampsia.

Dependent variable: severe preeclampsia

\section{Level of IL-10 in serum}

Study data demonstrated that in pregnant women with pregnancy complicated by preeclampsia, the serum concentration of antiinflammatory IL10 is confirmed as a significant predictor of the occurrence of severe preeclampsia. Increased serum concentrations of IL10 for one $\mathrm{pg} / \mathrm{mL}$ reduces the likelihood of development of severe preeclampsia by $89.6 \%$ ( $95 \%$ CI $0.016-0.678)$. The sensitivity of this parameter as a predictor for severe pre-eclampsia is $96 \%$, and the specificity is $80 \%$ (Table 13 ).

Analysis of the relationships between serum maternal concentration of IL10 and serum concentration of enzyme LDH, creatinine, platelets, proteinuria, and uric acid was also made.

The obtained values of Pearson's coefficients indicate negative correlations of IL10 with LDH and proteinuria, whereas the correlations of IL10 with creatinine, platelets, and uric acid were positive. However, significant correlations were confirmed only between IL10 and platelets as well as between IL10 and proteinuria. The correlation with the platelets count was positive which means that significantly higher concentration of IL10 was confirmed in patients with higher number of platelets in the blood, and vice versa. The correlation between IL10 and proteinuria was negative showing that the serum concentration of IL10 was significantly lower in patients with higher amount of proteins in the urine, and vice versa.

This study demonstrates differences in IL10 levels in women with preeclampsia compared to the levels in women with a normal pregnancy outcome. We found that in pregnant women with preeclampsia, the increased serum concentrations of IL10 predicted lower likelihood for the development of severe preeclampsia.

\section{Discussion}

One of the aim of our study was to define the demographic and socio-economic characteristics of pregnant women with the risk of pre-eclampsia in Macedonia. So as for this part of the study, the results 
Citation: Markova AD, Dimitrov G, Tofoski G, Georgievska J, Dzikova E, et al. (2019) Improving the Predictive Accuracy for Pre-eclampsia by Combining Demographic and Socio-economic Risk Factors with Biochemical Indicators. Int J Pediatr Neonat Care 5: 148. doi: https://doi.org/10.15344/2455-2364/2019/148

Page 6 of 6

showed that elevated systolic blood pressure of $160 \mathrm{mmHg}$ or higher, diastolic blood pressure of $100 \mathrm{mmHg}$ or higher, pregnancy at older age than 35 years as is nulliparity are associated with highly significant risk for developing severe form of pre-eclampsia. Other risk factors examinated in this survey such as duration of gestation, BMI, number of pregnencies, previous pregnancy with pre-eclampsia, diabetes and smoking status according to the results of this study, are risk factors that insignificantly increase the risk for severe form of pre-eclapmsia.

Next, knowing that the pregnancy is a condition that requires immunological tolerance and knowing that it is widely accepted that immune mechanisms are involved in pathogenesis of pregnancy complications such as pre-eclampsia, we examinated the correlation between IL10 serum concentrations (as a biochemical marker) and pre-eclamsia with its severity. Previous studies showed that in pregnancy complicated by preeclampsia, cytokine levels essentially change compared with the respective levels in physiological pregnancy. Thus, even a moderate form of preeclampsia shows directional change, i.e., elevated levels of pro- and anti-inflammatory cytokines, with the exception of IL-10, wherein a downward trend in severe preeclampsia is recorded.

\section{Results}

The regression analysis applied in this study showed that elevated systolic blood pressure of $160 \mathrm{mmHg}$ or higher, diastolic blood pressure of $100 \mathrm{mmHg}$ or higher, pregnancy at older age than 35 years as is nulliparity are associated with highly significant risk for developing severe form of pre-eclampsia. While other variables predicted higher likelihood for the development of severe preeclampsia, IL10 decreased such likelihood. IL10 was also found to be negatively correlated with proteinuria, and positively correlated with blood platelets. Significantly higher concentration of IL10 was confirmed in patients with higher number of platelets in the blood, and vice versa. On the other hand, the serum concentration of IL10 was significantly lower in patients with higher amount of proteins in the urine, and vice versa.

The actual study demonstrated platelets count and proteinuria as significant predictors of serum IL10 concentration - platelets count predicting higher serum concentration of IL10, while urine proteins predicting lower serum IL10.

\section{Conclusion}

Management of preeclampsia centers on early recognition and timely intervention to prevent serious morbidity and mortality. Despite recent advances in our understanding of the etiology of preeclampsia, there is still no clinically useful screening test. Since prevention of pre-eclampsia is not possible, the target should be to estimate the severity of the disease that will provide intensive supervision during the further course of pregnancy. That can be done by identifying risk factors that are associated with significant risk for developing severe form of pre-eclampsia and early recognition of the biochemical markers whose fluctuations also correlate with the risk for developing severe form of this disease.

\section{Competing Interests}

The authors declare that they have no competing interests.

\section{References}

1. Quan LM, Xu QL, Zhang GQ, Wu LL, Xu H, et al. (2018) An analysis of the risk factors of preeclampsia and prediction based on combined biochemical indexes. KJMS 34: 109-112.

2. Rosser ML, Katz NT (2013) Pre-eclampsia: an obstetrician's perspective. Adv Chronic kidney Dis 10: 287-276.

3. Raighupathy R (2013) Cytokines as key players in the pathophysiology of preeclampsia. Med Print Pract 22: 8-19.

4. Niljayan MV, Karunmanchi SA (2013) New developments in the pathogenesis of preeclampsia. Adv Chronic kidney Dis 20: 265-270.

5. Tsatsaris V, Fournier T, Winer N (2008) Pathophysiology of preeclampsia. J Gynecol Obstet Biol Reprod 1: 16-23.

6. Lewis DF, Canzoneri BJ, Wang Y (2009) Maternal circulating TNF-alpha levels are highly correlated with IL-10 levels, but not IL- 6 and IL- 8 levels, in women with preeclampsia. Am J Reprod Immunol 5: 269-274.

7. Sukhih GT, Vanko LV (2012) Immune factors in the etiology and pathogenesis of pregnancy complications. Obstetrics and Gynecology 1: 128-136.

8. Makarov IO, Shemanaeva TV, Hasanov SR, Popova OP (2009) Preeclampsia as a manifestation of immune endotheliosis. The Questions of Gynecology Obstetrics and Perinatology 6: 17-22.

9. Tosun M, Celik H, Avci B, Yavuz E, Alper T, et al. (2010) Maternal and umbilical serum levels of interleukin-6, interleukin-8, and tumor necrosis factor-alpha in normal pregnancies and in pregnancies complicated by Preeclampsia. J Matern Fetal Neonatal Med 23: 880-886.

10. Bosio PM, McKenna PJ, Conroy R, O'Herlihy C (1999) Maternal central hemodynamics in hypertensive disorders of pregnancy. Obstetric Gynecollogy 94: 978-984.

11. Hashii K, Fujiwara S, Yoshioka N, Kataoka S, Yamada T, et al. (1998) Peripheral blood mononuclear cells stimulate progesterone production by luteal cells derived from pregnant and nonpregnant women: possible involvement of interleukin-4 and interleukin-10 in corpus luteum function and differentiation. Hum Reprod 13: 2738.

12. Piccinni MP, Giudizi G, Biagotti R, Beloni L, Giannarini L, et al. (1995) Progesterone favors the development of human $T$ helper cells producing Th2 type cytokines and promotes both IL- 4 production and membrane CD30 expression in established Th1 cell clones. J Immunol 155: 128.

13. Chen BA, Parviainen K, Jeyabalan A (2008) Correlation of catheterized and clean catch urine protein/creatinine ratios in preeclampsia evaluation. Obstet Gynecol 112:606-610.

14. Khazardoost S, Abdollahi A Shafaat M (2012) Comparison of 8-h urine protein and random urinary protein-to-creatinine ratio with 24-h urine protein in pregnancy. J Matern Fetal Neonatal Med 25: 138.

15. McCrae KR (2006) Thrombocytopenia in Pregnancy in Platelets.

16. Ciantar E, Walker JJ (2011) Preeclampsia, Severe Preeclampsia and Hemolysis, Elevated Liver Enzymes and Low Platelets Syndrome. Women's Health 7: 555-569.

17. Côté AM, Firoz T, Mattman A (2008) The 24-hour urine collection: gold standard or historical practice. Am J Obstet Gynecol 199: 625.

18. Makarov IO, Shemanaeva TV, Hasanov SR, Popova OP (2009) Preeclampsia as a manifestation of immune endotheliosis. The Questions of Gynecology Obstetrics and Perinatology 6: 17-22. 